\title{
Eating behaviours, dietary profile and body composition according to dieting history in men and women of the Québec Family Study
}

\author{
Véronique Provencher ${ }^{1}$, Vicky Drapeau ${ }^{2}$, Angelo Tremblay $^{2}$, Jean-Pierre Després ${ }^{1,3}$, \\ Claude Bouchard ${ }^{4}$ and Simone Lemieux ${ }^{1 *}$ \\ ${ }^{1}$ Department of Food Science and Nutrition, Laval University, Ste-Foy, Québec, Canada, G1K 7P4 \\ ${ }^{2}$ Division of Kinesiology, Department of Preventive and Social Medicine, Laval University, Ste-Foy, Québec, \\ Canada \\ ${ }^{3}$ Québec Heart Institute, Laval Hospital Research Center, Laval University, Ste-Foy, Québec, Canada \\ ${ }^{4}$ Pennington Biomedical Research Center, Louisiana State University, Baton Rouge, Louisiana, USA
}

(Received 5 August 2003 - Revised 19 December 2003 - Accepted 3 February 2004)

\begin{abstract}
The purpose of the present cross-sectional study was to compare eating behaviours (cognitive dietary restraint, disinhibition and susceptibility to hunger), dietary profile and physiological variables according to the practice of dieting: current dieting; history of dieting in the 10-year period that preceded the study; no dieting during the same period. Dieting history, anthropometric markers of adiposity, RMR, dietary profile ( $3 \mathrm{~d}$ food record) and eating behaviours (three-factor eating questionnaire) were determined in a sample of 244 men and 352 women. A greater proportion of women $(31.8 \%)$ than men $(16.8 \%)$ reported that they had been on a diet over the past 10 years $(P=0.0001)$. In both genders, current and past dieters had a higher BMI $(P<0.05)$ than non-dieters and current dieters had lower reported energy intakes than past dieters and non-dieters (only in women) $(P<0 \cdot 05)$. Current and past dieters also had higher scores for all eating behaviours and their subscales $(P<0 \cdot 05$; except for susceptibility to hunger in men) compared with non-dieters (adjusted for age, reported energy intake, percentage of dietary fat, BMI and RMR). Moreover, for each dieting-history category, women had significantly higher scores for cognitive dietary restraint than men $(P<0.05)$. In conclusion, the present study showed that current and past dieters had higher scores for cognitive dietary restraint and disinhibition compared with non-dieters. As disinhibition has previously been associated with a greater risk of subsequent weight gain, interventions aimed at preventing an increase in disinhibition may be promising for longterm weight maintenance.
\end{abstract}

Dieting history: Three-factor eating questionnaire: Dietary patterns: Gender differences

A high proportion of individuals express the desire to lose weight in industrialised countries. For example, in the province of Québec (Canada), $53 \%$ of women and $34 \%$ of men would like to lose weight (Ledoux \& Rivard, 1998). Nevertheless, the prevalence of obesity is steadily increasing (World Health Organization, 1997), which suggests that most individuals do not successfully achieve their weight-loss goals. Studies have shown that long-term weight-loss maintenance following hypoenergetic diets is low, with only about 20 to $30 \%$ of overweight and obese individuals able to maintain their weight loss after 1 to 3 years and an even lower percentage after 3 to 5 years (Golay et al. 1997; Miller, 1999; Wing \& Hill, 2001). Moreover, more women want to lose weight than men (Ledoux \& Rivard, 1998), even if a greater proportion of men $(32 \%)$ than women $(23 \%)$ are overweight (BMI $\geq 27 \mathrm{~kg} / \mathrm{m}^{2}$; Ledoux \& Rivard, 1998).

It has been proposed that dieting may not always produce health benefits (Wooley \& Garner, 1991). In this regard, Doucet et al. (2001) observed that women with a history of dieting were more prone to weight gain than women who had never been on a diet. In addition, Korkeila et al. (1999) noted that frequent dieting was positively related to subsequent major weight gain. Studies have suggested that behavioural factors, such as cognitive dietary restraint and disinhibition, could contribute to weight regain in dieters (Karlsson et al. 1994; Fedoroff et al. 1997; Le Barzic, 2001). Thus, eating behaviours may be related to the history of dieting and gender differences could be present, but more investigations are needed to shed light on these issues.

In this regard, even if it is well established that women have a higher prevalence of dieting than men, there are nevertheless some men who are dieting to lose weight. Since few studies have been done in both men and women to compare their eating behaviours, dietary patterns and anthropometric profile according to the history of dieting, it appeared to be important to assess the presence of 
gender differences. Moreover, it has been recently shown that subscales for cognitive dietary restraint, disinhibition and susceptibility to hunger, as described by Westenhoefer et al. (1999) and Bond et al. (2001), are of particular interest for their refined definitions representing distinct aspects of these eating behaviours. It is also interesting to note that cross-sectional studies have previously shown negative associations between cognitive dietary restraint and susceptibility to hunger as well as positive relationships for disinhibition with rigid restraint and susceptibility to hunger (Westenhoefer et al. 1999; Provencher et al. 2003). However, gender differences, obesity status and dieting history could modulate these relationships. To our knowledge, little research has examined subscales for cognitive dietary restraint, disinhibition and susceptibility to hunger, particularly in relation to dieting history. Furthermore, dieting behaviours have been usually measured with general questions and without precision about a time period. In the present study, we have specified a time frame in the questions asked to the participants and we have particularly questioned them about their behaviour (dieting in order to lose weight) and not about their intention (desire to lose weight). Finally, dieters identified in this sample were dieting on their own, which means that they were not enrolled in a controlled weight-loss programme. This is an interesting aspect of the present study since dietary patterns, eating behaviours and physiological profile in this subgroup of the population have not been well documented.

The main purpose of the present cross-sectional study was therefore to compare eating behaviours (cognitive dietary restraint, disinhibition and susceptibility to hunger), dietary profile and physiological variables according to the history of dieting in both men and women. Subjects were classified into three exclusive categories of dieting history, which were: (1) currently dieting; (2) not currently dieting but with a history of dieting over the 10-year period preceding the study (past dieters); (3) not currently dieting with no history of dieting over the 10 -year period that preceded the study (non-dieters).

\section{Methods}

\section{Subjects}

The Québec Family Study (QFS) was initiated at Laval University in 1978 (Bouchard, 1996). The subjects were recruited through the media and were all French Canadians. Participation was voluntary and all subjects signed an informed consent document. The results presented in the present paper are derived from cross-sectional analyses of subjects who completed a questionnaire on eating behaviours (three-factor eating questionnaire; TFEQ). This questionnaire has been administered since 1993 and a subsample of the QFS subjects ( $n$ 279) completed the TFEQ for the first time while they were involved in the second phase of the QFS (1989 to 1998). The remaining subjects ( $n$ 317) completed the questionnaire for the first time while they were in the third phase of the QFS (1998 to 2002). Only data (dietary profile and physiological variables) collected at the moment of the first completion of the TFEQ were used for the present report. Moreover, for a given subject, all variables used in the present study were measured within a 1-month interval. The QFS was approved by the medical ethics committee of Laval University.

\section{Three-factor eating questionnaire}

A French version of the fifty-one-item TFEQ was selfadministrated to 596 healthy adults (244 men and 352 women) from the QFS and was analysed by the same observer. The purpose of this questionnaire is to assess three factors related to cognitions and behaviours associated with eating. These factors are cognitive dietary restraint (intent to control food intake), disinhibition (overconsumption of food in response to cognitive or emotional cues), and susceptibility to hunger (food intake in response to feelings and perceptions of hunger) (Stunkard \& Messick, 1985). This questionnaire has been validated and its three scales have been reported to show good test-retest reliability (Stunkard \& Messick, 1985; Laessle et al. 1989; Lluch, 1995).

More specific subscales for these three general eating behaviours have been proposed by Westenhoefer et al. (1999) and Bond et al. (2001). Cognitive dietary restraint has been divided into rigid control (dichotomous, all-ornothing approach to eating, dieting and weight), and flexible control (gradual approach to eating, dieting and weight) (Westenhoefer et al. 1999). Disinhibition has also been divided into three subscales: habitual susceptibility to disinhibition (occurring when circumstances could predispose to recurrent disinhibition); emotional susceptibility to disinhibition (associated with negative affective states); situational susceptibility to disinhibition (initiated by specific environmental cues) (Bond et al. 2001). Finally, susceptibility to hunger has also been divided into two specific subscales: internal locus for hunger (interpreted and regulated internally) and external locus for hunger (triggered by external cues) (Bond et al. 2001).

\section{Physiological profile and dietary patterns}

Body weight and height were measured according to standardised procedures (The Airlie (VA) Consensus Conference, 1988) and BMI was calculated. As previously reported (Doucet et al. 2001), RMR was assessed in the fasting state by indirect calorimetry measurements, performed over a $30 \mathrm{~min}$ period by using an open-circuit ventilated-hood system (Amatek 3-3A $\left(\mathrm{CO}_{2}\right.$ analyser) and Amatek CD-3A $\left(\mathrm{O}_{2}\right.$ analyser); Thermox Instruments Division, Pittsburgh, PA, USA). Subjects also quantified foods and drinks consumed using a $3 \mathrm{~d}$ estimated food record, which included two week days and one weekend day (Tremblay et al. 1983). Nutrient intakes were calculated with a computerised version of the Canadian Nutrient File (Health \& Welfare Canada, 1991). In order to take into account the possibility of under-reporting, cut-off limits were used, as proposed by Goldberg et al. (1991). These cut-off limits have been developed to identify subjects with a reported energy intake below $1.35 \times \mathrm{BMR}$, since 
such an intake cannot be representative of long-term habitual intakes. As described earlier (p. 998), a measurement of RMR was performed for all subjects involved in the present study. Although the RMR value for a given subject is generally slightly higher than their BMR value, a recent study has shown that the Schofield equations overestimate BMR value and, thus, this predicted BMR is unsuitable, especially among obese populations (Horgan \& Stubbs, 2003). Accordingly, in our sample, predicted BMR was slightly higher than measured RMR (data not shown). For all these reasons, it was decided to use RMR in our calculations since this value was actually measured, whereas BMR would have been estimated. Therefore, in our sample, subjects who had reported energy intakes that fell below $1.35 \times$ RMR were excluded from the analyses involving dietary variables. However, this exclusion criterion was applied only for past dieters and non-dieters, since current dieters may have lower energy intakes due to their actual dieting behaviours. The percentage of energy $(\mathrm{kJ})$ derived from proteins, carbohydrates and dietary fat was also calculated.

Each participant completed a questionnaire on general dietary patterns. Two questions were of particular interest in the context of the present study. Subjects had to indicate if they were currently dieting: 'Are you currently on a diet in order to lose weight?'. Subjects who answered 'yes' to this question were identified as 'current dieters'. Subjects also had to indicate whether they experienced dieting over the past 10 years: 'Have you been on a diet over the past 10 years?'. Those who did and who were not currently dieting were identified as 'past dieters'. Finally, subjects who did not report any dieting episode during the 10-year period that preceded the study, and who were not currently dieting, were identified as 'non-dieters'.

\section{Statistical analysis}

Percentages of current dieters, past dieters and non-dieters were compared between men and women using a $\chi^{2}$ test. In both genders, ANOVA were performed to assess the group effect on physical characteristics, dietary profile and eating behaviours. The Duncan multiple-range test was used to compare means between the three groups when a significant effect was observed. As significant associations between eating behaviours, anthropometric variables and dietary profile have been previously reported (Carmody et al. 1995; Lawson et al. 1995; Lindroos et al. 1997; Westenhoefer et al. 1999; Provencher et al. 2003), it was decided to control for the potential confounding effect of these variables on eating behaviours. Analysis of covariance, which combines regression analysis with ANOVA, statistically removes the variation in the dependent variable that is associated with the variation in the covariate, which must be a source of variation believed to affect the dependent variable (Kirk, 1982). Thus, a covariance analysis was performed to evaluate differences in eating behaviours between current dieters, past dieters and non-dieters, in both men and women after controlling for age, reported energy intake, percentage of energy derived from dietary fat, BMI and RMR. In the presence of a significant effect, multiple comparisons were performed using the 'LSMEANS' procedure. The probability level for significance used for the interpretation of all statistical analyses was set at an $\alpha$ level of $P<0 \cdot 05$. All analyses were performed using SAS statistical software (SAS Institute Inc., Cary, NC, USA).

\section{Results}

Overall, $8.2 \%$ of subjects were currently dieting, while $25.7 \%$ of subjects had experienced dieting over the last 10 -year period (past dieters). More specifically, Table 1 shows that a lower proportion of men $(16.8 \%)$ than women $(31.8 \%)$ were considered as past dieters $\left(\chi^{2} 17.0\right.$; $P=0.0001)$ whereas no gender differences were observed for the prevalence of current dieters. In women, past dieters and current dieters were not significantly different with regard to BMI while both groups of dieters (current and past) were characterised by a significantly higher BMI than non-dieters $(P<0.05)$. In men, past dieters had a

Table 1. Age, body mass index, resting metabolic rate and dietary profile of male and female current dieters, past dieters and non-dieters ${ }^{*}$ (Mean values and standard deviations)

\begin{tabular}{|c|c|c|c|c|c|c|c|c|c|c|c|c|}
\hline & \multicolumn{6}{|c|}{ Men } & \multicolumn{6}{|c|}{ Women } \\
\hline & \multicolumn{2}{|c|}{ Current dieters } & \multicolumn{2}{|c|}{ Past dieters } & \multicolumn{2}{|c|}{ Non-dieters } & \multicolumn{2}{|c|}{ Current dieters } & \multicolumn{2}{|c|}{ Past dieters } & \multicolumn{2}{|c|}{ Non-dieters } \\
\hline & Mean & $\overline{S D}$ & Mean & $\overline{S D}$ & Mean & $\overline{S D}$ & Mean & $\overline{S D}$ & Mean & $\overline{S D}$ & Mean & $\overline{S D}$ \\
\hline Subjects: $n$ & \multicolumn{2}{|c|}{$\begin{array}{l}17 \\
7.0\end{array}$} & \multicolumn{2}{|c|}{$\begin{array}{l}41 \\
16 \cdot 8\end{array}$} & \multicolumn{2}{|c|}{$\begin{array}{l}186 \\
76 \cdot 2\end{array}$} & \multicolumn{2}{|c|}{$\begin{array}{l}32 \\
9 \cdot 1\end{array}$} & \multicolumn{2}{|c|}{$\begin{array}{l}112 \\
31 \cdot 8\end{array}$} & \multicolumn{2}{|c|}{$\begin{array}{l}208 \\
59 \cdot 1\end{array}$} \\
\hline Age (years) & $46 \cdot 8^{a}$ & $13 \cdot 1$ & $45 \cdot 4^{a}$ & $12 \cdot 6$ & $42 \cdot 7^{a}$ & $15 \cdot 5$ & $45 \cdot 8^{\mathrm{a}}$ & $15 \cdot 0$ & $42 \cdot 3^{\mathrm{a}}$ & $13 \cdot 3$ & $41.2^{\mathrm{a}}$ & $15 \cdot 1$ \\
\hline BMI $\left(\mathrm{kg} / \mathrm{m}^{2}\right)$ & $31.0^{\mathrm{b}}$ & $5 \cdot 1$ & $37 \cdot 1^{\mathrm{a}}$ & 8.7 & $26 \cdot 4^{c}$ & $5 \cdot 0$ & $31.5^{\mathrm{a}}$ & 7.5 & $33 \cdot 7^{\mathrm{a}}$ & 9.7 & $25 \cdot 7^{b}$ & $5 \cdot 6$ \\
\hline RMR† (MJ) & $7.9^{a}$ & 1.7 & $8.4^{\mathrm{a}}$ & 1.7 & $6 \cdot 4^{\mathrm{b}}$ & 1.2 & $6 \cdot 0^{\mathrm{a}}$ & 0.9 & $6 \cdot 2^{\mathrm{a}}$ & 1.3 & $5 \cdot 3^{b}$ & 0.9 \\
\hline Energy intake (MJ) & $11.0^{\mathrm{a}}$ & $3 \cdot 8$ & $13 \cdot 2^{\mathrm{a}}$ & $3 \cdot 1$ & $12 \cdot 4^{\mathrm{a}}$ & $2 \cdot 8$ & $8 \cdot 1^{\mathrm{a}}$ & $2 \cdot 1$ & $9 \cdot 7^{b}$ & $2 \cdot 2$ & $9 \cdot 3^{b}$ & 1.9 \\
\hline Proteins (\%)‡ & $17 \cdot 3^{\mathrm{a}}$ & $4 \cdot 2$ & $16 \cdot 1^{\mathrm{a}}$ & $2 \cdot 8$ & $15 \cdot 4^{\mathrm{a}}$ & $2 \cdot 8$ & $19 \cdot 2^{\mathrm{a}}$ & $4 \cdot 2$ & $16 \cdot 2^{b}$ & 3.2 & $15 \cdot 8^{\mathrm{b}}$ & 2.9 \\
\hline Dietary fat $(\%) \ddagger$ & $33 \cdot 1^{a}$ & $7 \cdot 7$ & $37 \cdot 3^{\mathrm{b}}$ & 4.9 & $33 \cdot 7^{\mathrm{a}}$ & $6 \cdot 0$ & $34 \cdot 3^{\mathrm{ab}}$ & 8.4 & $36 \cdot 4^{\mathrm{b}}$ & 5.5 & $33 \cdot 8^{a}$ & 5.6 \\
\hline Carbohydrates (\%)‡ & $47 \cdot 3^{a}$ & 9.4 & $44 \cdot 8^{\mathrm{a}}$ & 5.5 & $48 \cdot 1^{\mathrm{a}}$ & $7 \cdot 0$ & $45 \cdot 6^{\mathrm{a}}$ & 7.9 & $45 \cdot 1^{a}$ & 6.7 & $48 \cdot 6^{\mathrm{b}}$ & $5 \cdot 9$ \\
\hline
\end{tabular}

a,b,c Mean values with unlike superscript letters are significantly different $(P<0.05)$

* Analyses were conducted in men and women separately. Dietary variables are reported only for past dieters and non-dieters with valid energy intakes, i.e. for 183 men and 240 women, while all current dieters are included in these analyses. 
higher BMI than current dieters and non-dieters $(P<0.05)$. $\mathrm{BMI}$ was also higher in current dieters than in non-dieters $(P<0.05)$. In both genders, RMR was significantly lower in non-dieters than in current dieters and past dieters $(P<0.05)$, whereas no differences were observed when values were adjusted for BMI (data not shown). Regarding reported energy intake, no differences were observed for this variable in men whereas women who were currently dieting had lower reported energy intake than past dieters and non-dieters $(P<0 \cdot 05)$. With regard to macronutrient intake distribution, past dieters had a significantly higher proportion of energy derived from fat than non-dieters $(P<0.05)$ in both genders. Moreover, in women, current dieters had a higher proportion of energy derived from protein than past dieters and non-dieters $(P<0.05)$ as well as a lower proportion of energy derived from carbohydrates than non-dieters $(P<0 \cdot 05)$.

As indicated earlier (p. 999), eating behaviours were associated with age, reported energy intake, percentage of energy derived from dietary fat, BMI and RMR. It was therefore decided to compare eating behaviours according to dieting history after controlling for the potential confounding effect of these variables on eating behaviours. Accordingly, Fig. 1 shows that, in men, current dieters and past dieters had higher scores than non-dieters for cognitive dietary restraint $(P<0 \cdot 05)$. No differences in disinhibition were observed between current dieters and past dieters as well as between current dieters and non-dieters. However, men who were past dieters had significantly higher scores for disinhibition than non-dieters $(P<0.05)$. In women (Fig. 2), current dieters had higher scores for cognitive dietary restraint and disinhibition than past dieters $(P<0.05)$ and past dieters had higher scores for cognitive dietary restraint and disinhibition than non-dieters $(P<0 \cdot 05)$. While no differences were observed for susceptibility to hunger and its subscales by dieting history in men (Fig. 1), women who were currently dieting had

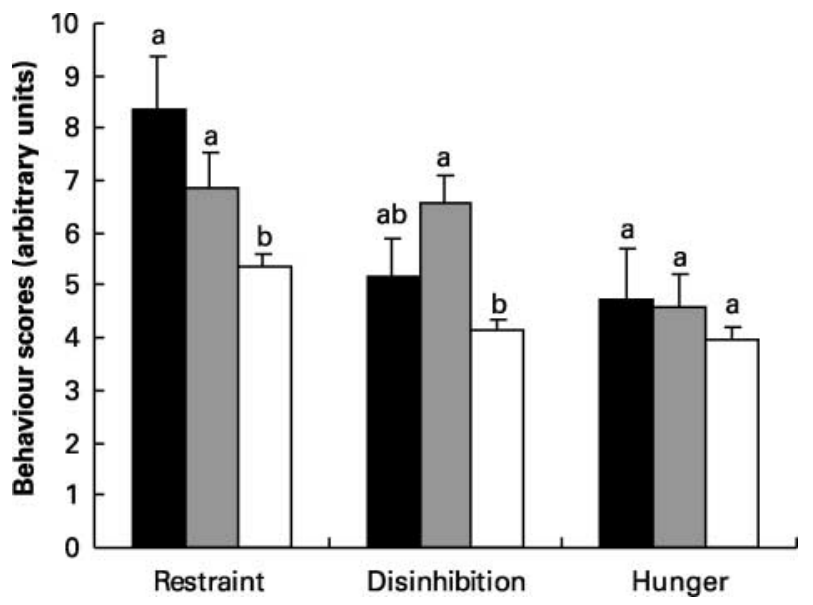

Fig. 1. Differences in eating behaviours between current dieters $(\square ; n$ 17), past dieters $(\square ; n 41)$ and non-dieters $(\square ; n 186)$ in men. Values are means and are adjusted for age, reported energy intake, percentage of energy derived from dietary fat, BMI and RMR. Standard errors are represented by vertical bars. Values within each eating behaviour with unlike superscript letters are significantly different $(P<0.05)$.

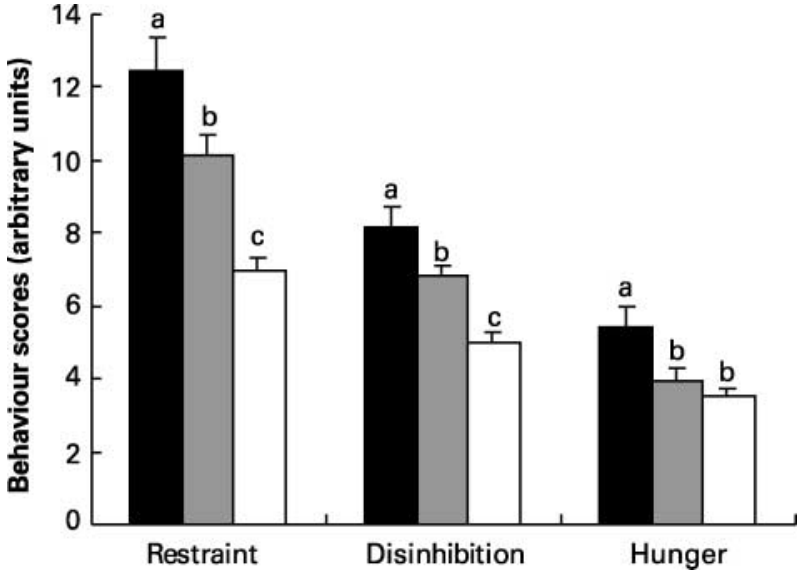

Fig. 2. Differences in eating behaviours between current dieters ( $\square$; $n$ 32), past dieters ( $\square$; $n$ 112) and non-dieters $(\square ; n$ 208) in women. Values are means and are adjusted for age, reported energy intake, percentage of energy derived from dietary fat, BMI and RMR. Standard errors are represented by vertical bars. Values within each eating behaviour with unlike superscript letters are significantly different $(P<0.05)$.

significantly higher scores for susceptibility to hunger than past dieters and non-dieters (Fig. 2).

Figs. 3 and 4 show differences in the subscales of cognitive dietary restraint and disinhibition according to dieting history after adjustment for confounding variables. As observed in Fig. 3, men who were current dieters had higher scores for flexible restraint than non-dieters $(P<0.05)$. Rigid restraint was significantly higher in men who were current dieters or past dieters than in non-dieters $(P<0.05)$. Habitual susceptibility to disinhibition was higher for past dieters than for current and non-dieters $(P<0.05)$. Emotional susceptibility to disinhibition was also higher for men who were past dieters than for nondieters $(P<0 \cdot 05)$, whereas current dieters were not significantly different from past dieters and non-dieters for this variable. No differences were noted between past dieters,

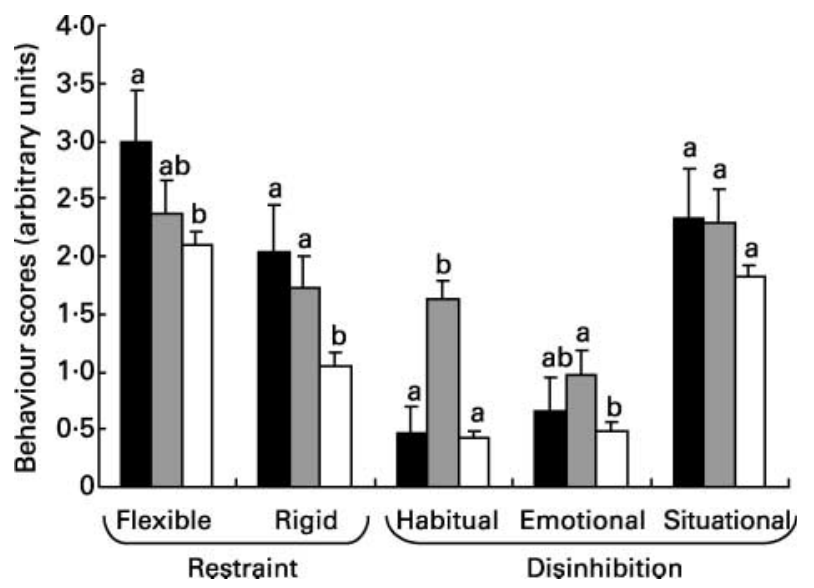

Fig. 3. Differences in subscales for cognitive dietary restraint and disinhibition between current dieters $(\square ; n 17)$, past dieters $(\square ; n 41)$ and non-dieters ( $\square ; n$ 186) in men. Values are means and are adjusted for age, reported energy intake, percentage of energy derived from dietary fat, BMI and RMR. Standard errors are represented by vertical bars. Values within each eating behaviour with unlike superscript letters are significantly different $(P<0.05)$. 


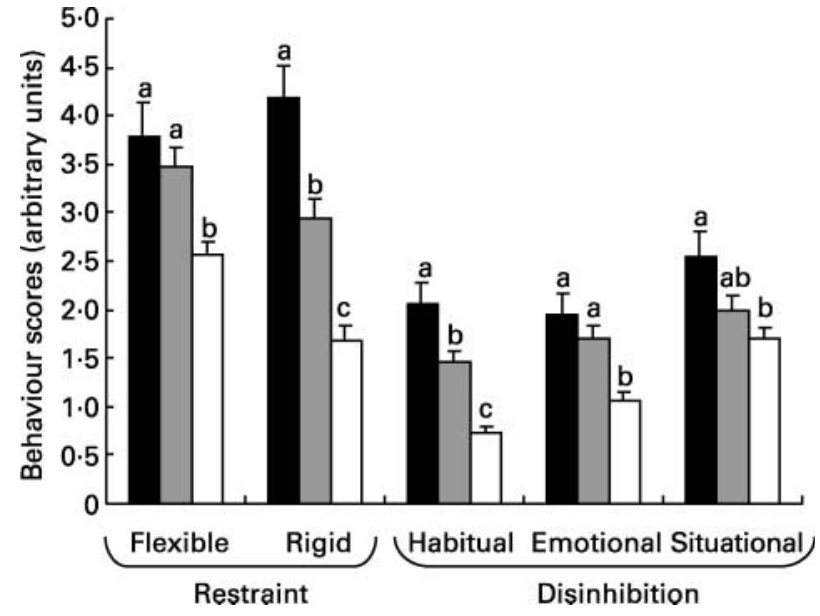

Fig. 4. Differences in subscales for cognitive dietary restraint and disinhibition between current dieters $(\square ; n 32)$, past dieters ( $\square$; $n$ 112) and non-dieters $(\square ; n$ 208) in women. Values are means and are adjusted for age, reported energy intake, percentage of energy derived from dietary fat, BMI and RMR. Standard errors are represented by vertical bars. Values within each eating behaviour with unlike superscript letters are significantly different $(P<0.05)$.

current dieters and non-dieters for situational susceptibility to disinhibition in men. As shown in Fig. 4, women who were currently dieting had higher scores for rigid restraint and habitual susceptibility to disinhibition than past dieters $(P<0.05)$, and past dieters had higher scores than non-dieters for those two subscales $(P<0 \cdot 05)$. Moreover, flexible restraint, as well as emotional susceptibility to disinhibition, was higher in current dieters and past dieters than in non-dieters $(P<0 \cdot 05)$. While no differences were observed between current dieters and past dieters as well as between past dieters and non-dieters, women who were currently dieting had significantly higher scores than non-dieters for situational susceptibility to disinhibition (Fig. $4 ; P<0.05$ ) as well as for the two subscales of susceptibility to hunger (internal hunger and external hunger) (data not shown; $P<0 \cdot 05$ ).

For each category of dieting history, women had consistently higher scores for cognitive dietary restraint than men $(P<0.05)$. Among current dieters, women had significantly higher scores for disinhibition than men $(P<0.05)$, while no gender difference was observed for disinhibition among past dieters. Among non-dieters, women had significantly higher scores for disinhibition than men $(P<0.05)$. Finally, in all three dieting history categories, no gender differences were noted for susceptibility to hunger.

\section{Discussion}

The main purpose of the present study was to compare eating behaviours (cognitive dietary restraint, disinhibition and susceptibility to hunger), dietary profile and physiological variables according to dieting history in both men and women. The present results showed that a higher proportion of women than men had been dieting over the 10year period that preceded the study, a finding concordant with previous literature (Carmody et al. 1995; NeumarkSztainer et al. 1999). In both genders, subjects who were currently dieting or who dieted in the past had a higher BMI than subjects who were considered as non-dieters. The present results are consistent with other findings showing that overweight and obese individuals will more often attempt to lose weight than those who are not overweight (French \& Jeffery, 1994; Ledoux \& Rivard, 1998).

The present data also showed that, in women, current dieters had lower reported energy intakes than past dieters and non-dieters (a trend is observed in men; $P=0.08$ ), suggesting that individuals on a weight-loss diet are in fact eating less to create a negative energy balance. However, the possibility of under-reporting in current dieters cannot be excluded, since we could not apply cut-off limits for under-reporting in these subjects, who probably have, in fact, a lower energy intake than energy expenditure because of their current dieting status. Among past dieters, the results showed that even if men and women had higher RMR values than non-dieters, no differences were observed regarding reported energy intakes between past dieters and non-dieters. Accordingly, when reported energy intakes were divided by RMR, a significant difference was observed between past dieters and non-dieters, in both genders $(1.45(\mathrm{SD} 0.36) v .1 .72(\mathrm{SD} 0.42)$ in men and 1.43 (SD 0.42) v. 1.68 (SD 0.42) in women; $P<0.05$ ). This means that past dieters had lower reported energy intakes compared with their energy needs. To explain this issue, it may be possible that past dieters had a lower energy expenditure, since they could have a lower physical activity level than non-dieters. Accordingly, in our sample, a trend was observed for non-dieters to have a higher daily energy expenditure from moderate to vigorous physical activity compared with past dieters (data not shown; $P=0.06$ ). The relatively lower reported energy intake for given energy needs observed in past dieters may also be partly explained by the possibility of underreporting. Although the validity of reported energy intake was taken into account in the present study, since past dieters and non-dieters who reported implausibly low energy intakes compared with their energy needs (Goldberg et al. 1991) were excluded from the analyses involving dietary variables, there is still a possibility that past dieters may have under-reported their energy intake. For example, subjects who report consuming more than $1.35 \times \mathrm{RMR}$ can still be under-reporting their intakes. Moreover, this phenomenon is especially present among obese subjects (Goris et al. 2000) as well as in those who have higher scores for cognitive dietary restraint (Bathalon et al. 2000), which are two characteristics that described well the past dieters of our sample.

With regard to dietary profile, men and women who were currently dieting or who had never been on a diet had a macronutrient intake distribution meeting nutritional recommendations (Institute of Medicine \& Food and Nutrition Board, 2002). However, individuals who had been on a diet over the preceding 10 years had a higher proportion of energy derived from dietary fat than the recommended 25 to $35 \%$ of energy, which suggests that the dieting experience may not foster better eating habits.

Many significant differences in eating behaviours were observed according to dieting history in both genders. Because associations between age, reported energy 
intake, percentage of energy derived from dietary fat, BMI, RMR and eating behaviours were observed, we decided to perform analyses with control over these variables and found that significant differences remained in eating behaviours between current dieters, past dieters and non-dieters. Therefore, differences in eating behaviours could not be explained by obesity nor by age, reported energy intake, percentage of energy derived from dietary fat, BMI and RMR. It is interesting to emphasise that we observed higher scores for cognitive dietary restraint as well as higher scores for disinhibition (only in women) in current dieters than in non-dieters. Higher cognitive dietary restraint has been consistently reported in current dieters compared with non-dieters (Karlsson et al. 1994; Foster et al. 1998), whereas higher disinhibition is not a consistent finding among current dieters. The present results could be explained by the fact that our subjects were not enrolled in a controlled weight-loss intervention programme in which food available to subjects is restricted to that offered. Since a lot of food choices were available consistently to these subjects in a real-life uncontrolled context, it may be easier for them to overeat in response to cognitive or emotional cues.

From the present results, it is not possible to determine whether it is dieting that alters eating behaviours or whether some specific eating behaviours predispose to dieting. In this regard, some studies have evaluated eating behaviours before and after dieting episodes and have demonstrated that dieting may induce significant changes in eating behaviours (Karlsson et al. 1994; Foster et al. 1998). After a weight-loss treatment, Karlsson et al. (1994) and Foster et al. (1998) found that cognitive dietary restraint scores increased while disinhibition and susceptibility to hunger scores decreased. These studies suggest that dieting behaviours may have an effect on eating behaviours, but the long-term effect of dieting on eating behaviours remains to be established. On the other hand, it has been recently proposed that eating behaviours may be partly explained by familial resemblance (Provencher et al. 2002; Steinle et al. 2002). Moreover, even if other studies are needed to confirm this finding, the linkage of particular chromosomal regions to eating-behaviour phenotypes has been observed in the Amish population (Steinle et al. 2002) as well as in the QFS (Bouchard et al. 2003). Thus, the present results suggest that specific eating behaviours may be present before episodes of dieting, which could explain the predisposition of these individuals to dieting behaviours or to weight regain.

Past dieters had generally higher scores than non-dieters for cognitive dietary restraint, disinhibition and their subscales. The present results could suggest that higher scores for these eating behaviours persist even after the end of a diet. It is interesting to note that a positive relationship between disinhibition and cognitive dietary restraint has been reported previously (Lawson et al. 1995; Williamson et al. 1995; Lindroos et al. 1997; Provencher et al. 2003). Studies have also shown that restrained eaters were more sensitive and reactive to food and emotional cues. They ate significantly more than unrestrained eaters after sensory or cognitive exposures to food as well as to positive or negative mood states (Eldredge, 1993; Fedoroff et al. 1997; Wardle et al.
2000; Bellisle \& Dalix, 2001), which could be related to a higher expression of disinhibition. According to Heatherton et al. (1988) disinhibition would be a consequence of higher cognitive dietary restraint and the present results support this hypothesis since past dieters displayed higher scores than non-dieters for both cognitive dietary restraint and disinhibition. Thus, it could also be proposed that disinhibition is critical when it comes to the predisposition of individuals to regain weight. Furthermore, in a free-living context, it has been reported that women who had a higher score for cognitive dietary restraint were at a higher risk for weight gain since they could not maintain their high restraint behaviour over time (Drapeau et al. 2003). Therefore, lowering disinhibition directly or indirectly through a reduction in restraint behaviours could contribute to prevent further weight gain.

As indicated earlier (p. 1000), the pattern of differences in disinhibition and its subscales according to dieting history was not the same in men and women. In women, current and past dieters consistently displayed higher scores than non-dieters for disinhibition and all its subscales (habitual, emotional and situational susceptibility to disinhibition). In men, past dieters showed significantly higher scores than non-dieters for disinhibition as well as for habitual and emotional susceptibility to disinhibition, whereas no differences were observed between current dieters and non-dieters for disinhibition and its subscales. The present results suggest that there is a gender difference in the association between disinhibition and dieting history. While they are currently dieting, men seem to be less prone to disinhibition than women, since their scores are similar to those displayed by non-dieters. Previous studies have shown that women display higher scores for cognitive dietary restraint, flexible restraint and rigid restraint than men (Carmody et al. 1995; Provencher et al. 2003). It has been suggested that higher scores for cognitive dietary restraint in women would be explained by the fact that women are more likely to be on a diet than men. In the present study, we found that among subjects who were non-dieters, women still had higher scores for cognitive dietary restraint and its subscales than men. Thus, the more frequent dieting episodes among women did not totally explain the higher cognitive dietary restraint, flexible restraint and rigid restraint scores compared with men, since women who were not dieting still had higher scores for cognitive dietary restraint than men. Therefore, accordingly to de Castro (1995) it could be possible that women who are not dieting express restrained behaviours in response to greater awareness and concern about food and fear of gaining weight, whereas men usually express restrained behaviours in order to lose weight. By consistently exerting restriction over their food intake, it may be difficult for women to continuously maintain this level of cognitive dietary restraint, which may result in more frequent episodes of overeating that translate into higher disinhibition scores.

In conclusion, the present results showed that current and past dieters had generally higher scores for cognitive dietary restraint and disinhibition than non-dieters. Enhancing cognitive dietary restraint, which is often the focus of weight-loss intervention, may not be optimal for 
the long-term control of body weight, since it could result in higher scores for disinhibition, which are predictive of weight gain (Hays et al. 2002). Thus, the present results suggest that alternative strategies that would prevent an increase in disinhibition may be promising for the longterm maintenance of a reduced body weight.

\section{Acknowledgements}

V. P. is a recipient of a studentship from the Fonds de la Recherche en Santé du Québec and S. L. is a research scholar from the Fonds de la Recherche en Santé du Québec. The authors express their gratitude to the subjects for their excellent collaboration and the staff of the Physical Activity Sciences Laboratory for their contribution to the present study. We especially thank Dr G. Thériault, L. Allard, G. Bouchard, S. Brulotte, L. Bargone, M. Chagnon, G. Fournier, H. Bessette and C. Leblanc for their help in the collection and analysis of the data. The QFS has been supported over the years by multiple grants from the Medical Research Council of Canada and other Canadian agencies. The present paper was supported by the Canadian Institutes of Health Research (MGC-15187).

\section{References}

Bathalon GP, Tucker KL, Hays NP, Vinken AG, Greenberg AS, McCrory MA \& Roberts SB (2000) Psychological measures of eating behavior and the accuracy of 3 common dietary assessment methods in healthy postmenopausal women. Am J Clin Nutr 71, 739-745.

Bellisle F \& Dalix AM (2001) Cognitive restraint can be offset by distraction, leading to increased meal intake in women. Am J Clin Nutr 74, 197-200.

Bond MJ, McDowell AJ \& Wilkinson JY (2001) The measurement of dietary restraint, disinhibition and hunger: an examination of the factor structure of the three factor eating questionnaire (TFEQ). Int J Obes Relat Metab Disord 25, 900-906.

Bouchard C (1996) Genetic and body fat content. In Progress in Obesity Research, pp. 33-41 [A Angel, H Anderson, C Bouchard, D Lau, L Leither and R Mendelson, editors]. London, UK: John Libbey.

Bouchard L, Drapeau V, Provencher V, Bouchard C, Lemieux S \& Pérusse L (2003) Genome-wide scan analysis of eating behaviors in the Québec Family Study (QFS). Presented at the 2nd Conference of the International Society for Behavioral Nutrition and Physical Activity. Abstr. www.isbnpa.org/ Meeting_2003/Saturday_\%20oral\%20abstracts.pdf

Carmody TP, Brunner RL \& St Jeor ST (1995) Dietary helplessness and disinhibition in weight cyclers and maintainers. Int $J$ Eat Disord 18, 247-256.

de Castro JM (1995) The relationship of cognitive restraint to the spontaneous food and fluid intake of free-living humans. Physiol Behav 57, 287-295.

Doucet E, St-Pierre S, Almeras N, Despres JP, Bouchard C \& Tremblay A (2001) Evidence for the existence of adaptive thermogenesis during weight loss. Br J Nutr 85, 715-723.

Drapeau V, Provencher V, Lemieux S, Despres JP, Bouchard C \& Tremblay A (2003) Do 6-y changes in eating behaviors predict changes in body weight? Results from the Quebec Family Study. Int J Obes Relat Metab Disord 27, 808-814.

Eldredge KL (1993) An investigation of the influence of dieting and self-esteem on dietary disinhibition. Int J Eat Disord 13, $57-67$.
Fedoroff IC, Polivy J \& Herman CP (1997) The effect of preexposure to food cues on the eating behavior of restrained and unrestrained eaters. Appetite 28, 33-47.

Foster GD, Wadden TA, Swain RM, Stunkard AJ, Platte P \& Vogt RA (1998) The eating inventory in obese women: clinical correlates and relationship to weight loss. Int $J$ Obes Relat Metab Disord 22, 778-785.

French SA \& Jeffery RW (1994) Consequences of dieting to lose weight: effects on physical and mental health. Health Psychol 13, 195-212.

Golay A, Hagon I, Painot D, Rouget P, Allaz AF, Morel Y, Nicolet J \& Archinard M (1997) Personalities and alimentary behaviors in obese patients. Patient Educ Couns 31, 103-112.

Goldberg GR, Black AE, Jebb SA, Cole TJ, Murgatroyd PR, Coward WA \& Prentice AM (1991) Critical evaluation of energy intake data using fundamental principles of energy physiology: 1. Derivation of cut-off limits to identify underrecording. Eur J Clin Nutr 45, 569-581.

Goris AH, Westerterp-Plantenga MS \& Westerterp KR (2000) Undereating and underrecording of habitual food intake in obese men: selective underreporting of fat intake. Am J Clin Nutr 71, 130-134.

Hays NP, Bathalon GP, McCrory MA, Roubenoff R, Lipman R \& Roberts SB (2002) Eating behavior correlates of adult weight gain and obesity in healthy women aged 55-65 y. Am J Clin Nutr 75, 476-483.

Health and Welfare Canada (1991) The Canadian Nutrient File Ottawa. Ontario, Canada: Health and Welfare Canada.

Heatherton TF, Herman CP, Polivy J, King GA \& McGree ST (1988) The (mis)measurement of restraint: an analysis of conceptual and psychometric issues. J Abnorm Psychol 97, 19-28.

Horgan GW \& Stubbs J (2003) Predicting basal metabolic rate in the obese is difficult. Eur J Clin Nutr 57, 335-340.

Institute of Medicine \& Food and Nutrition Board (2002) Dietary Reference Intakes for Energy, Carbohydrate, Fiber, Fat, Fatty Acids, Cholesterol, Protein, and Amino Acids (Macronutrients). A Report of the Panel on Macronutrients, Subcommittees on Upper Reference Levels of Nutrients and Interpretation and Uses of Dietary Reference Intakes, and the Standing Committee on the Scientific Evaluation of Dietary Reference Intakes, pp. 1-936. Washington, DC: National Academy of Sciences.

Karlsson J, Hallgren P, Kral Lindroos AK, Sjostrom L \& Sullivan M (1994) Predictors and effects of long-term dieting on mental well-being and weight loss in obese women. Appetite 23, $15-26$.

Kirk RE (1982) Analysis of covariance. In Experimental Design: Procedures for the Behavioral Sciences, 2nd ed., pp. 715-762 [Baylor University, editor]. Pacific Grove, CA: Brooks/Cole Publishing Company.

Korkeila M, Rissanen A, Kaprio J, Sorensen TI \& Koskenvuo M (1999) Weight-loss attempts and risk of major weight gain: a prospective study in Finnish adults. Am J Clin Nutr 70, 965-975.

Laessle RG, Tuschl RJ, Kotthaus BC \& Pirke KM (1989) A comparison of the validity of three scales for the assessment of dietary restraint. J Abnorm Psychol 98, 504-507.

Lawson OJ, Williamson DA, Champagne CM, DeLany JP, Brooks ER, Howat PM, Wozniak PJ, Bray GA \& Ryan DH (1995) The association of body weight, dietary intake, and energy expenditure with dietary restraint and disinhibition. Obes Res 3, 153-161.

Le Barzic M (2001) The syndrome of cognitive restraint: from the nutritional standard to eating disorders (in French). Diabetes Metab 27, 512-516.

Ledoux M \& Rivard M (1998) Poids corporel. In Enquête sociale et de santé 1998. www.stat.gouv.qc.ca/publications/sante/ e_soc-sante98_pdf.htm 
Lindroos AK, Lissner L, Mathiassen ME, Karlsson J, Sullivan M, Bengtsson C \& Sjostrom L (1997) Dietary intake in relation to restrained eating, disinhibition, and hunger in obese and nonobese Swedish women. Obes Res 5, 175-182.

Lluch A (1995) Identification des conduites alimentaires par approches nutritionnelles et psychométriques: implications thérapeutiques et préventives dans l'obésité humaines. PhD thesis, Université Henri Poincaré, Nancy I, France.

Miller WC (1999) How effective are traditional dietary and exercise interventions for weight loss? Med Sci Sports Exerc 31, $1129-1134$.

Neumark-Sztainer D, Sherwood NE, French SA \& Jeffery RW (1999) Weight control behaviors among adult men and women: cause for concern? Obes Res 7, 179-188.

Provencher V, Drapeau V, Tremblay A, Despres JP \& Lemieux S (2003) Eating behaviors and indexes of body composition in men and women from the Québec Family Study. Obesity Res 11, 783-792.

Provencher V, Pérusse L, Drapeau V, Tremblay A, Després JP \& Lemieux S (2002) Familial resemblance in eating behaviors in men and women from the Québec Family Study. J Am Diet Assoc 102, A-35, Abstr.

Steinle NI, Hsueh WC, Snitker S, Pollin TI, Sakul H, St Jean PL, Bell CJ, Mitchell BD \& Shuldiner AR (2002) Eating behavior in the Old Order Amish: heritability analysis and a genomewide linkage analysis. Am J Clin Nutr 75, 1098-1106.
Stunkard AJ \& Messick S (1985) The three-factor eating questionnaire to measure dietary restraint, disinhibition and hunger. J Psychosom Res 29, 71-83.

The Airlie (VA) Consensus Conference (1988). In Standardization of Anthropometric Measurements, pp. 39-80. [T Lohman, A Roche and R Martorel, editors]. Champaign, IL: Human Kinetics Publishers.

Tremblay A, Sévigny J, Leblanc C \& Bouchard C (1983) The reproducibility of a three-day dietary record. Nutr Res 3, 819-830.

Wardle J, Steptoe A, Oliver G \& Lipsey Z (2000) Stress, dietary restraint and food intake. J Psychosom Res 48, 195-202.

Westenhoefer J, Stunkard AJ \& Pudel V (1999) Validation of the flexible and rigid control dimensions of dietary restraint. Int $J$ Eat Disord 26, 53-64.

Williamson DA, Lawson OJ, Brooks ER, Wozniak PJ, Ryan DH, Bray GA \& Duchmann EG (1995) Association of body mass with dietary restraint and disinhibition. Appetite $\mathbf{2 5}$, $31-41$.

Wing RR \& Hill JO (2001) Successful weight loss maintenance. Annu Rev Nutr 21, 323-341.

Wooley SC \& Garner DM (1991) Obesity treatment: the high cost of false hope. J Am Diet Assoc 91, 1248-1251.

World Health Organization (1997) Obesity: Preventing and Managing the Global Epidemic. Report on a WHO consultation on obesity. Geneva, Switzerland: WHO. 